\title{
Entrapment of the Temporal Horn as a Cause of Pure Wernicke Aphasia: Case Report
}

\author{
Aldo Spallone $^{1,2}$ Daniele Belvisi ${ }^{3} \quad$ Luca Marsili $^{1,4}$ \\ 1 Section of Neurosurgery, Department of Clinical Neurosciences, \\ Neurological Centre of Latium (NCL), Rome, Italy \\ 2 Department of Biomedicine, University of Rome “Tor Vergata," \\ Rome, Italy \\ ${ }^{3}$ Neuromed Institute (IRCCS), Pozzilli (IS), Italy \\ ${ }^{4}$ Department of Neurology and Psychiatry, "Sapienza” University of \\ Rome, Viale dell'Università, Rome, Italy \\ J Neurol Surg Rep 2015;76:e109-e112.
}

\author{
Address for correspondence Aldo Spallone, MD, Neurological Centre \\ of Latium (NCL), Via Patrica 15, Rome, Italy \\ (e-mail: aldospallone@hotmail.com).
}

\author{
Abstract \\ Keywords \\ - entrapment of \\ temporal horn \\ - dominant hemisphere \\ - Wernicke aphasia \\ - successful ventricular \\ diversion
}

Entrapment of the temporal horn is an extremely rare pathologic condition occurring as a result of surgery for tumors, intraventricular infections, hemorrhage, or traumatic events involving the peritrigonal area. We report a case of a 58-year-old man who presented with pure Wernicke aphasia (never described before in the albeit rare cases of isolated temporal horn dilatation) that regressed completely following successful ventriculoperitoneal shunting. The relevant literature is also briefly reviewed.

\section{Introduction}

Isolated dilatation of the temporal horn ("entrapped temporal horn") is a rare pathologic condition and a management challenge. A very thoughtful review of the literature in 2013 reported only 24 cases. $^{1}$

We report an additional case of this extremely rare pathologic entity that appears to be unique for two reasons: the quite atypical clinical presentation, a pure sensory aphasia that the patient himself was able to describe perfectly as soon as he recovered normal speech function, and a very long follow-up of almost 10 years.

\section{Case Report}

A 58-year-old man was admitted to our institution in June 2004 with a brief complaint of sensory aphasia. The patient could walk and did not show signs of motor weakness; however, sensory function and visual fields could not be evaluated. A preadmission noncontrast computed tomography scan showed an isolated dilatation of the left temporal horn and signs of previous intracranial surgery. In fact he had been admitted a few weeks earlier to another neurosurgical unit where surgery was carried out for "intraventricular tumor" and a subsequent postoperative infection that had been managed successfully. The patient was single and had no relatives, and no further information could be obtained regarding his recent past clinical history. General examination at admission was unremarkable. Magnetic resonance imaging (MRI) (-Fig. 1) showed dilatation of the left temporal horn with a suspiciously abnormal area at the level of the trigone, which showed no if little enhancement, however, with contrast ( $\mathbf{F}$ Fig. 1). Due to the diagnostic suspicion of an intraventricular tumor, an intraventricular approach via a left portion parietal paramedian approach was performed, at which a virtually abnormal tissue was apparently totally removed from the left trigonal area and an internal temporal-to-frontal shunt system was positioned using a Pudenz ventricular catheter. Histologic examination showed only signs of tissue scarring received

November 26, 2014

accepted

January 13, 2015

published online

May 13, 2015
DOI http://dx.doi.org/

10.1055/s-0035-1549225. ISSN 2193-6366. (c) 2015 Georg Thieme Verlag KG
Stuttgart . New York

License terms

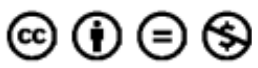




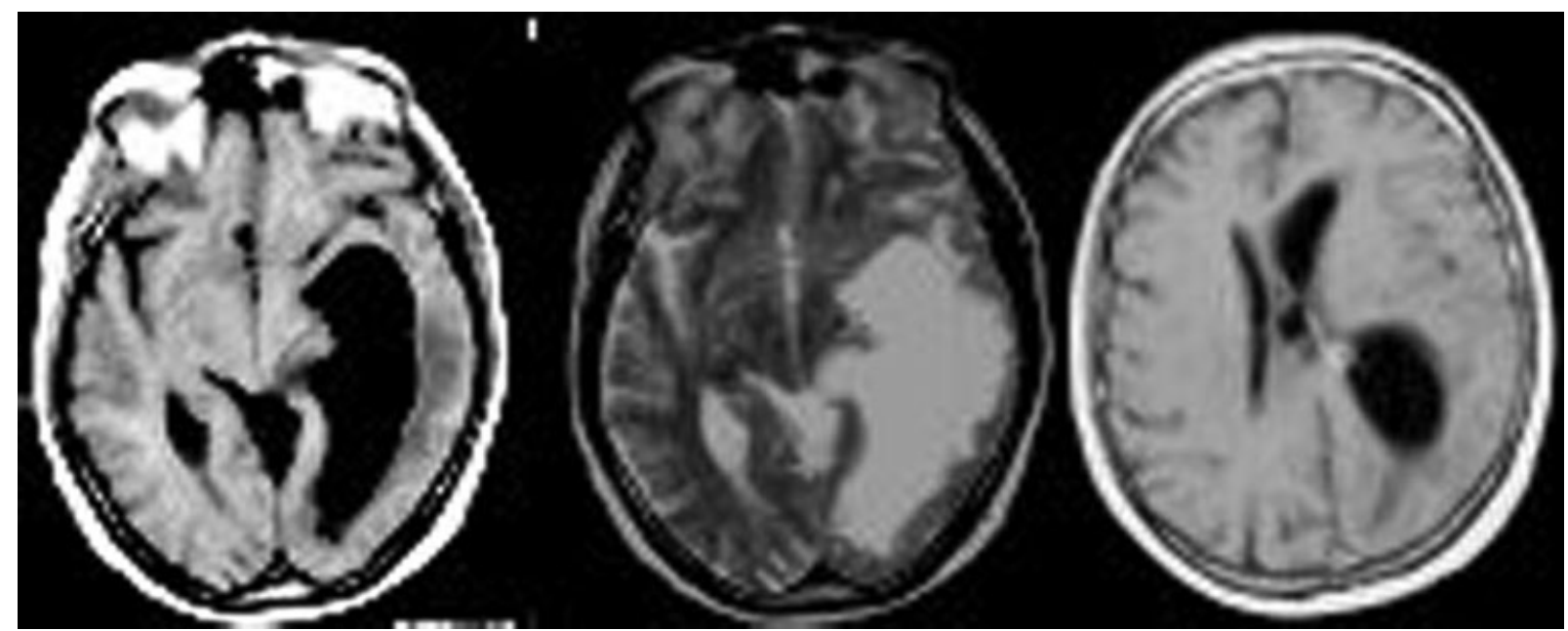

Fig. 1 Preoperative magnetic resonance imaging. Left panel, T1 image, axial view: The examination shows a significant dilatation of a temporal horn, with slight midline shift and flattened sulci on the left side. Center panel: T2 sequence gives evidence of perilesional edema involving the left temporal area. Right panel: Postcontrast T1 image shows slight enhancement at the level of the medial wall of the dilated ventricle.

with reactive gliosis and signs of local parenchymal hemorrhage (-Fig. 2).

However, the clinical symptoms did not improve, and a control MRI performed 9 days after surgery showed persistent temporal horn dilatation. Therefore a ventriculoperitoneal (VP) shunt was inserted on the next day, after which his aphasia steadily improved. The patient was discharged home 25 days following admission with no more speech deficits. He was able to remember most of the time when he had been aphasic and described his situation as "being in China where everybody spoke an unintelligible language and nobody could understand me." He spoke fluently but with incomprehensible, often nonsense, words and was not able to understand and/or follow any commands. Later on he was able to resume his previous job as a tailor, when he subsequently left due to a form of depression that he had complained about earlier. He remained clinically stable and underwent serial imaging

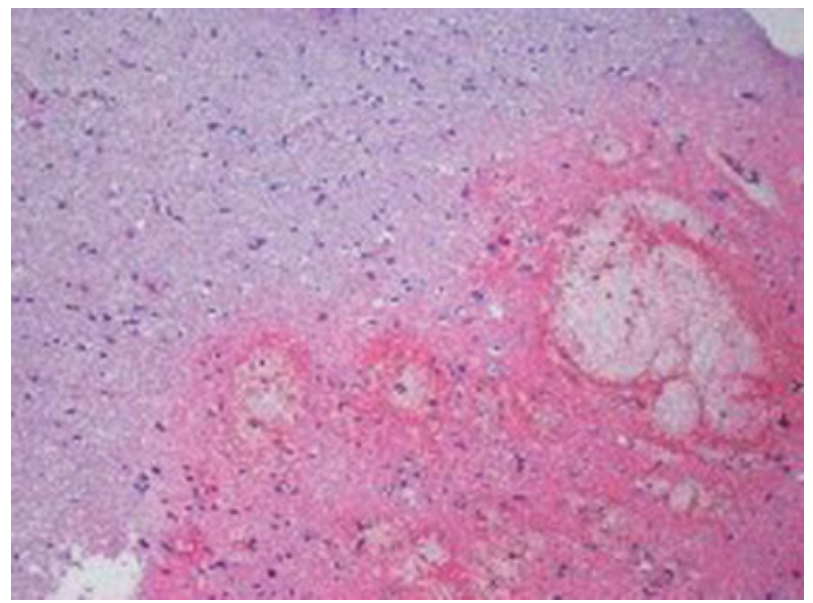

Fig. 2 Micrograph of cerebral tissue biopsy shows evidence of edema, intraparenchymal hemorrhage, and reactive inflammation with macrophagic infiltration. Hematoxylin and eosin stain; original magnification $\times 100$. follow-ups (-Figs. 3 and 4), the last of which, in 2013 ( - Fig. 5), showed no enlargement of the shunted temporal horn.

\section{Discussion}

Entrapment of the temporal horn can be related to several pathologic conditions including paraventricular tumors, infections, hemorrhages, and traumatic (penetrating) lesions. $^{2-8}$ All these pathologic conditions could cause an obstruction of the cerebrospinal fluid flow at the level of the trigone, as a rule related to scarring of the ventricular ependyma.

\section{Review of the Literature}

Of the 23 cases in which patient age was specified, 17 were adults, ${ }^{2-12} 6$ of whom were $>50$ years of age. ${ }^{2,6,7}$ of these, eight ${ }^{2,3,7,8}$ had entrapment of the temporal horn that developed in relationship to a para-intraventricular tumor and not infrequently as a consequence of its incomplete removal. $3,7,8,12$ In five cases an intraventricular infection was the cause. ${ }^{3,4,6,10}$ Neurosarcoidosis represented the causative factor in two instances. ${ }^{11,12}$ In the remaining case only scarring due to the previous removal of a peritrigonal arteriovenous malformation led to subsequent entrapment of the temporal horn. ${ }^{3}$

\section{Present Case}

In the present case we were not able to determine with absolute certainty the original cause of the ependymal scarring causing the entrapment of the left temporal horn. In fact, clinical data on the previous admission in another hospital were impossible to obtain, and the patient, single with no relatives living in our city, was obviously not helpful in this respect. We performed an intraventricular exploration as a first step when the MRI was suspicious for an intraventricular tumor but found only histologically confirmed signs of local 


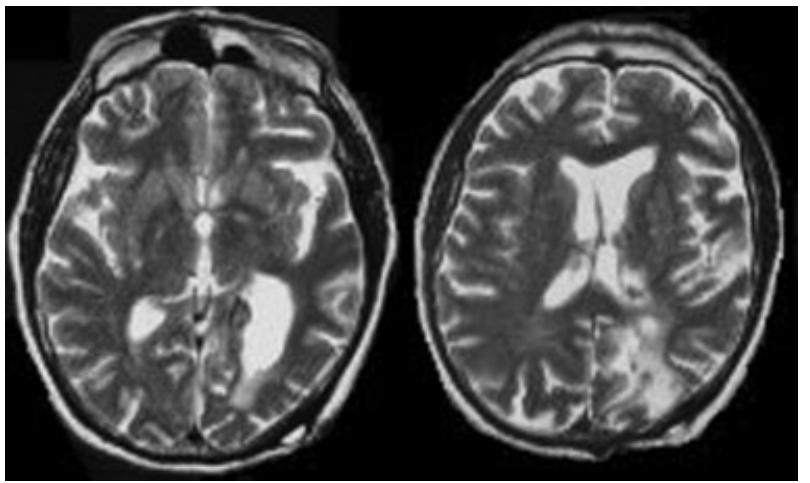

Fig. 3 Early postoperative magnetic resonance imaging T2 image following ventriculoperitoneal shunt, axial view. Left panel: Preoperative dilatation of the left temporal horn is significantly reduced and edema is no longer present, as is the preoperative shift. Right panel: The surgical access to the ventricle is demonstrated.

scarring (from previous surgery? from infection?). A temporal-to-frontal internal shunt was unsuccessful, and a few days later a VP shunt was inserted that proved to be effective and led to total regression of the patient's clinical symptoms.

The clinical presentation of our patient was in fact unique. Pure Wernicke aphasia has never been reported in cases of entrapment of the temporal horn. The patient spoke fluently but with either incomprehensible or illogical words and was unable to obey (and to understand) any command, but he could recall many episodes of his hospital stay when he recovered speech function. As noted earlier, he described his condition as being "in China surrounded by people who could not understand him at all."

\section{Surgical Technique}

The lack of information regarding previous treatment and the doubtful indications of the patient's preoperative diagnostic imaging led us to perform an open exploration. The VP shunt eventually led to a definitive success that persisted in the long term. Modern endoscopic techniques with fenestration of the trapped portions of the ventricular system are a sound

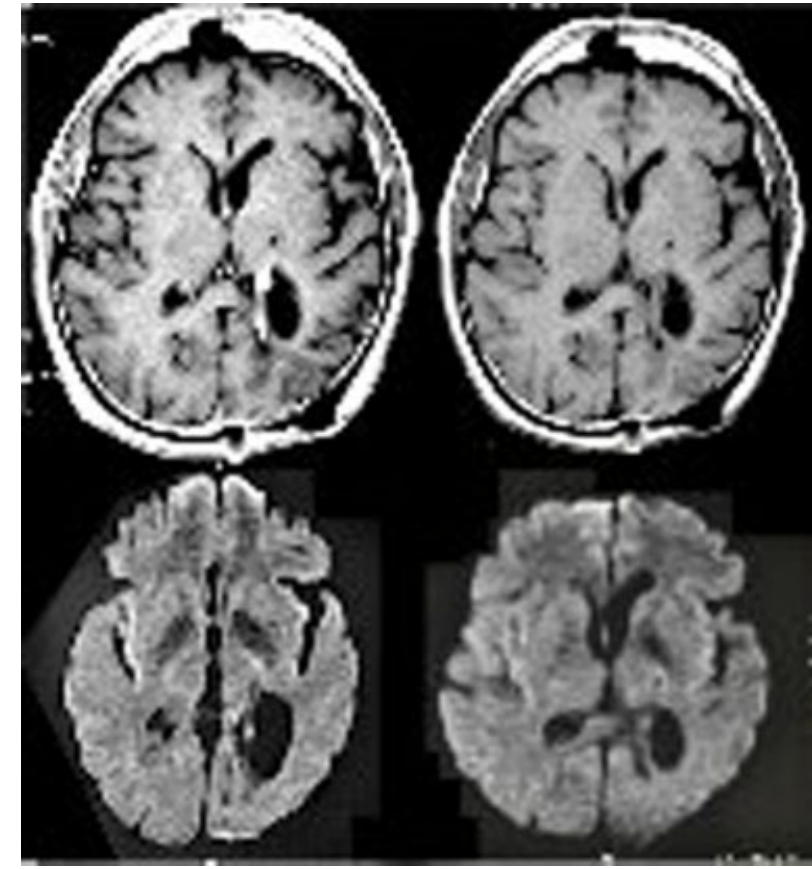

Fig. 4 One-year magnetic resonance imaging (MRI) control. Left upper panel: Postcontrast MRI shows enhancement of the medial ventricular wall as in the preoperative scan. Right upper panel: $\mathrm{T} 1$ axial view shows almost normal appearance of the ventricular system with just a slight dilatation of the temporal and frontal horns. Lower panels: Diffusion-weighted imaging does not show abnormalities except for modest ventricular dilatation.

alternative and possibly the treatment of choice in the near future. However a recent paper ${ }^{1}$ clearly stressed that such procedures are not at all risk free and must be performed with extreme caution and the necessary experience. Also, its impact in the long term is not known.

In conclusion, we reported a case of pure Wernicke aphasia occurring as a result of entrapment of the left temporal horn. Resolution of the clinical symptoms followed VP shunting and persisted in the long term.

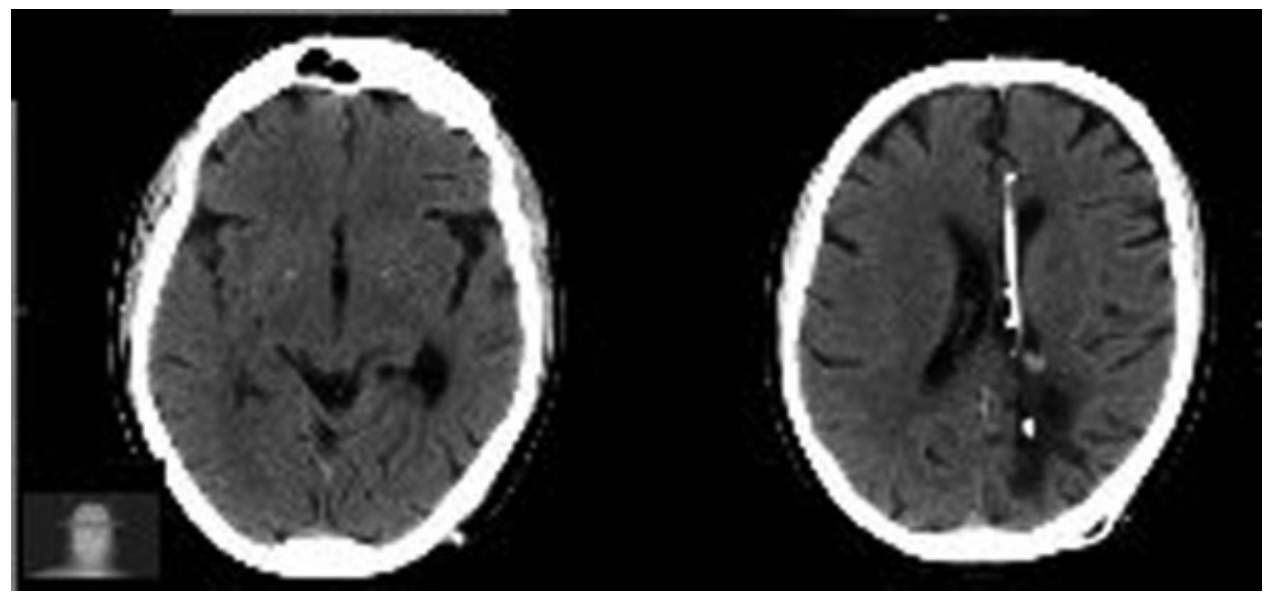

Fig. 5 Nine-year control computed tomography (CT) scan. Left panel: CT scan shows normal appearance of the brain except for a slightly dilated left temporal horn. Right panel: The higher cut shows a ventricular catheter and the surgical approach. 
e112 Entrapment of the Temporal Horn Spallone et al.

\section{References}

1 Krähenbühl AK, Baldauf J, Gaab MR, Schroeder HW. Endoscopic temporal ventriculocisternostomy: an option for the treatment of trapped temporal horns. J Neurosurg Pediatr 2013;11(5):568-574

2 Smith H, Moody D, Ball M, Laster W, Kelly DLJr, Alexander EJr. The trapped temporal horn: a trap in neuroradiological diagnosis. Neurosurgery 1979;5(2):245-249

3 Maurice-Williams RS, Choksey M. Entrapment of the temporal horn: a form of focal obstructive hydrocephalus. J Neurol Neurosurg Psychiatry 1986;49(3):238-242

4 Tsugane R, Shimoda M, Yamaguchi T, Yamamoto I, Sato O. Entrapment of the temporal horn: a form of focal non-communicating hydrocephalus caused by intraventricular block of cerebrospinal fluid flow -report of two cases. Neurol Med Chir (Tokyo) 1992;32(4):210-214

5 Watanabe T, Katayama Y. Evaluation by magnetic resonance imaging of the entrapped temporal horn syndrome. J Neurol Neurosurg Psychiatry 1999;66(1):113

6 Parrent AG. Endoscopically guided fenestration of the choroidal fissure for treatment of trapped temporal horn. J Neurosurg 2000; 93(5):891-894
7 Hervey-Jumper SL, Ziewacz JE, Heth JA, Sullivan SE. Frontal-totemporal horn shunt as treatment for temporal horn entrapment. J Neurosurg 2010;112(2):410-413

8 Chen CC, Kasper EM, Zinn PO, Warnke PC. Management of entrapped temporal horn by temporal horn to prepontine cistern shunting. World Neurosurg 2013;79(2):404.e7-404.e10

9 Cairns H, Daniel P, Johnson RT, Northcroft GB. Localized hydrocephalus following penetrating wounds of the ventricle. Br J Surg 1947;55(Suppl 1):187-197

10 Ofori-Kwakye SK, Wang AM, Morris JH, O'Reilly GV, Fischer EG, Rumbaugh CL. Septation and focal dilatation of ventricles associated with cryptococcal meningoencephalitis. Surg Neurol 1986; 25(3):253-260

11 Schlitt M, Duvall ER, Bonnin J, Morawetz RB. Neurosarcoidosis causing ventricular loculation, hydrocephalus, and death. Surg Neurol 1986;26(1):67-71

12 Berhouma M, Abderrazek K, Krichen W, Jemel H. Apropos of an unusual and menacing presentation of neurosarcoidosis: the space-occupying trapped temporal horn. Clin Neurol Neurosurg 2009;111(2):196-199 